\title{
Spontaneous Onset of Torsade de Pointes in Long-QT Syndrome and the Role of Sympathovagal Imbalance
}

\author{
Akira Fujiki, MD; Kunihiro Nishida, MD; Koichi Mizumaki, MD; \\ Hidehiko Nagasawa, MD; Mayumi Shimono, MD; Hiroshi Inoue, MD
}

\begin{abstract}
The net effects of sympathetic and vagal activity on the QT interval and the mode of spontaneous onset of torsade de pointes (TdP) are still unclear in long-QT syndrome. Two patients with long-QT syndrome had syncope while undergoing Holter ECG investigation. The spontaneous onset of TdP in these patients was analyzed with respect to the relation between the RR and QT intervals. Both patients were high-school students (16- and 17-year-old boys) who had been diagnosed as long-QT syndrome and followed up without medical treatment because they had had neither a history of syncope nor arrhythmia induction by treadmill exercise tests. The first episode of syncope in both patients occurred during ordinary daily life and was not related to exercise or psychological stress. The dynamic changes between the RR and QT intervals associated with the spontaneous onset of TdP were analyzed by Holter ECG. Both patients showed sinus tachycardia followed by abrupt sinus bradycardia immediately before the onset of TdP. The enhanced rate of the adaptive response of the QT interval that occurred during the deceleration of the heart rate preceded the onset of TdP. These observations suggest that the complex situation that follows sympathovagal imbalance may have an important role in the dynamic change in the QT interval and initiation of TdP in patients with long-QT syndrome. (Jpn Circ J 2001; 65: 1087-1090)
\end{abstract}

Key Words: Autonomic nerves; Long-QT syndrome; Torsade de pointes

$\mathbf{I}$ n long-QT syndrome, most ventricular tachyarrhythmias are triggered when the QT interval is markedly prolonged because of enhanced sympathetic activity!,2 However, in normal subjects increased sympathetic tone induces sinus tachycardia, which consequently shortens the QT interval. The net effects of sympathetic activity on heart rate (HR) and the QT interval, and the mode of spontaneous onset of torsade de pointes (TdP) are still unclear in longQT syndrome $3^{3-5}$ In our previous study, we evaluated the relation between the QT interval and autonomic nervous activity in long-QT syndrome and found that during recurrent syncopal episodes, the relation of the QT interval with the RR interval and HR variability indices changed markedly and the significant correlation disappeared!

Despite the relatively high prevalence of long-QT syndrome, few patients have syncope while undergoing Holter ECG investigation, which would enable analysis of the period immediately preceding the spontaneous onset of TdP3,4 We present 2 patients who did have a syncopal episode while wearing a Holter ECG, enabling us to evaluate the dynamic relation between the RR and QT intervals preceding the spontaneous onset of $\mathrm{TdP}$.

\section{Methods}

\section{Measurement of the RR and QT Intervals}

From the 24-h Holter ECG recordings, the QT interval

(Received February 26, 2001; revised manuscript received April 13, 2001; accepted April 20, 2001)

The Second Department of Internal Medicine, Toyama Medical and Pharmaceutical University, Toyama, Japan

Mailing address: Akira Fujiki, MD, The Second Department of Internal Medicine, Toyama Medical and Pharmaceutical University, 2630 Sugitani, Toyama 930-0194, Japan. E-mail: fujiki@toyamampu.ac.jp was measured manually from the earliest deflection of the QRS complex to the end of the $T$ wave. The point of the maximum down slope of the $T$ wave crossing the isoelectric line was used as the end of the T wave, as in our previous study6 The corrected QT interval (QTc) was calculated by dividing the measured QT interval by the square root of the preceding RR interval in seconds using Bazett's formula. Before the onset of TdP we measured both the RR and QT intervals on a beat-to-beat basis. The difference in repeated measurements by 2 observers was less than $10 \mathrm{~ms}$.

\section{Results}

\section{Patient 1}

The patient was a 16-year-old senior high-school student with a history of prolonged QT interval since the age of 13 . The rest of his medical history was unremarkable and there was no family history of sudden cardiac death. The treadmill exercise test showed no ventricular arrhythmias and he was allowed to play sport. He was a member of a ski club and had never experienced syncope or dizziness during exercise. At the age of 16 , he had a syncopal attack at rest after dinner. The next day, he underwent 24-h Holter ECG monitoring, which showed an episode of $\mathrm{TdP}$ during talking with his friends in the evening.

The surface ECG showed a markedly prolonged QT interval of $0.52 \mathrm{~s}(\mathrm{QTc}$ interval $0.45 \mathrm{~s}$ ) and terminal T wave inversion in the precordial leads (Fig 1). A subtle bifid T wave with a second component on top of the $\mathrm{T}$ wave was recorded in the limb and left precordial leads. During the 20 s period before the onset of TdP, the RR interval shortened gradually from 1.08 to $0.62 \mathrm{~s}$ and reached the minimum value (HR acceleration phase) (Fig 2). The QT interval shortened from 0.56 to $0.50 \mathrm{~s}$ along with the shortening of the RR interval. In the period that immediately 

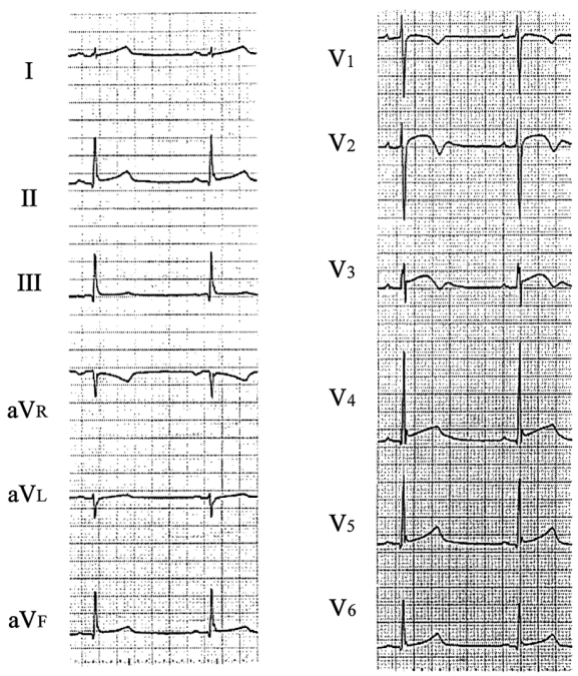

Fig 1. 12-lead ECG recording in patient 1 showing sinus bradycardia and markedly prolonged QT interval with terminal $\mathrm{T}$ wave inversion in the precordial leads.
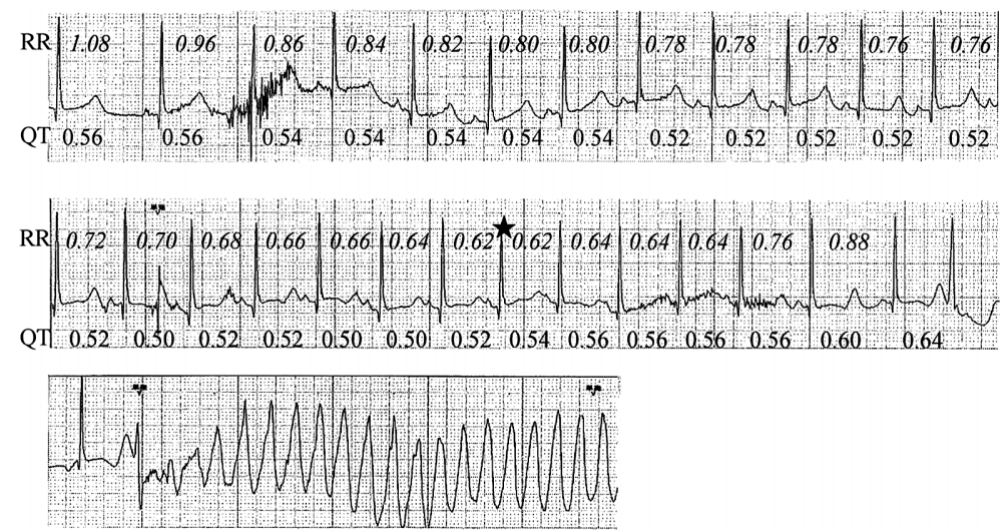

Fig 2. Initiation of torsade de pointes during Holter ECG recording in patient 1 . The RR interval shortens and reaches the minimum value $(\star)$ (acceleration phase). An abrupt increase in the RR interval, associated with the prolongation of QT interval, precedes the onset of torsades de pointes (deceleration phase). preceded the onset of TdP, there was an abrupt increase in the RR interval associated with a prolongation of the QT interval (the maximum QT interval of $0.64 \mathrm{~s}$ ) (HR deceleration phase). This marked prolongation of the QT interval was followed by a premature ventricular contraction that increased the coupling interval of the next sinus beat (compensatory pause). The pause was followed by the onset of TdP, a phenomenon of short-long-short sequence. The relation between the RR and QT intervals on a one-beat basis revealed that a gradual physiological shortening of the QT interval was followed by a marked QT prolongation along with an increase in the RR interval before the onset of TdP. The slope of the regression line of RR with QT during the deceleration phase $(0.38)$ became steeper than that during the acceleration phase $(0.15)$.

\section{Patient 2}

The patient was a 17-year-old senior high-school student with a diagnosis of prolonged QT interval at the age of 7. He had no family history of syncope or sudden cardiac death. When he entered senior high school he was examined using the Holter ECG and treadmill exercise test because of the prolonged QT interval, but ventricular arrhythmia was not detected and he was diagnosed as asymptomatic long-QT syndrome. He was allowed to play soccer. He had his first syncopal episode at the age of 17 while talking with his friends after he had played soccer, but had not previously experienced syncope during exercise.
The next day, a Holter ECG recording showed 2 episodes of TdP; one occurred in the evening while lying in the bed and the other was in the morning while sitting in a chair.

The surface ECG showed a prolonged QT interval of $0.52 \mathrm{~s}$ (QTc interval $0.47 \mathrm{~s}$ ) in the precordial leads (Fig 3). During the $30 \mathrm{~s}$ period preceding the syncope the RR interval shortened gradually from 1.20 to $0.60 \mathrm{~s}$ and reached the minimum value (HR acceleration phase) at 9 beats before the onset of TdP along with shortening of the QT interval from 0.62 to $0.46 \mathrm{~s}$ (Fig 4). Then, the RR interval increased abruptly and the prolongation of the QT interval (the maximum QT interval of $0.60 \mathrm{~s}$ ), associated with the notched T wave, preceded the onset of TdP (HR deceleration phase). The slope of the regression line of RR with QT during the deceleration phase $(0.53)$ became steeper than that during the acceleration phase $(0.29)$, similar to the findings in patient 1 .

In both patients during the 24-h Holter ECG monitoring, neither bradycardia-dependent abnormal prolongation of the QT interval nor T wave alternance was found. From the onset pattern of TdP and the notched $\mathrm{T}$ wave we speculate that both patients may have Ikr malfunction. They have been treated with propranolol $(>1 \mathrm{mg} / \mathrm{kg})$ for 2 years without recurrence of syncope.

\section{Discussion}

The first episode of syncope was not related to exercise 






Fig 3. 12-lead ECG recording in patient 2 showing sinus bradycardia and prolonged QT interval in the precordial leads.



Fig 4. Initiation of torsade de pointes during Holter ECG recording in patient 2 . The RR interval shortens from 1.20 to $0.72 \mathrm{~s}$ and the terminal portion of the $\mathrm{T}$ wave increases slightly (*). The RR interval shortens further from 0.72 to $0.60 \mathrm{~s}$ and reaches the minimum value $(\star)$ (acceleration phase) at 9 beats before the onset of torsade de pointes. Then, the RR interval increases abruptly and the prolongation of the QT interval (the maximum QT interval $0.60 \mathrm{~s}$ ), associated with the notched $\mathrm{T}$ wave, follows in the period that precedes the onset of torsades de pointes (deceleration phase). or psychological stress in either of the present patients. Both showed sinus tachycardia followed by abrupt sinus bradycardia immediately before the onset of TdP, which is compatible with simultaneous enhancement of sympathovagal activity before the onset of $\mathrm{TdP}$, and enhanced prolongation of the QT interval during the HR deceleration phase preceded the onset of TdP. These observations suggest that the complex situation that follows sympathovagal imbalance may have an important role in the dynamic change in the QT interval and the initiation of TdP in longQT syndrome.

\section{Onset of Torsades de Pointes}

The interactions between HR and sympathetic nervous activity are complex and may have some relation to the initiation of TdP? In long-QT syndrome the marked prolongation of the QT interval and the syncopal episodes are usually observed with enhanced sympathetic activity; that is, during physical exercise or psychological stress,, 2 The level of adrenergic drive required for the occurrence of TdP could also produce a sinus tachycardia that would prevent QT prolongation. Rate- and adrenergic-dependence of the QT interval may account for the absence of TdP during extreme sinus tachycardia in some patients? In patients with acquired long-QT syndrome, Locati et al reported that both adrenergic- and pause-dependent mechanisms may have a synergetic role in the genesis of $\mathrm{TdP}^{4}$ They demonstrated that an oscillatory short-long cycle length sequence preceded the onset of TdP and indicated that vagal tone activation was a possible trigger of cycle length oscillation.

Both patients presented here showed sinus tachycardia followed by the abrupt slowing of the sinus rate before the onset of TdP. The level of sympathetic activity may be still high at the onset of arrhythmia and the abrupt enhancement of vagal tone that slows the sinus rate may facilitate initiation of TdP as Locati et al suggested.

\section{QT Interval and Autonomic Nerve Activity}

Autonomic tone influences the QT interval directly by affecting the action potential duration and indirectly by modulating the RR interval. In normal subjects, vagal stimulation causes a rate-independent prolongation of the QT interval, whereas the exercise-induced increase in sympathetic tone causes a rate-independent shortening,8,9 In longQT syndrome 24-h Holter ECG recording during the syncope-free period reveals a positive correlation between the QT and RR intervals, but no significant correlation during recurrent syncopal episodes6 In the present study, the 
relation of the QT interval with the RR interval during the HR deceleration and acceleration phases changed remarkably and these variable relations may contribute to the different modulation of the QT interval by the HR during recurrent syncope ${ }^{10}$

Although the net autonomic effect on the sinus node (RR interval) remains constant, the net effect of sympathetic and vagal tone on the ventricle can be variable and induce differences in the QT interval, because of the predominance of sympathetic activity in the ventricle! ${ }^{1}$ Patients with long-QT syndrome show significant shortening of the QT interval after left cardiac sympathetic denervation, and it has been suggested that the spatial imbalance of sympathetic innervation may play a role in increasing the susceptibility to ventricular arrhythmias? In our previous study the QTc interval in healthy subjects failed to show significant changes after pharmacological right or left stellate ganglion block! ${ }^{2}$

In patients with long-QT1 and Iks malfunction, more than $80 \%$ are symptomatic during physical stress. However, patients with long-QT2 and Ikr malfunction become symptomatic with auditory stimuli and emotional stress $!^{13}$ Thus, in long-QT syndrome sympathetic modulation of the QT interval under specific conditions varies in a gene-specific manner. In both of the present patients, the ST-T wave pattern suggested Ikr malfunction, but we have not performed gene analysis.

\section{Clinical Implications}

Patients 1 and 2 were originally diagnosed with asymptomatic long-QT syndrome and were followed up without medical treatment. However, they had episodes of TdP during ordinary daily activities. After physical training the effect of vagally induced HR deceleration in the setting of increased sympathetic activity may have been accentuated. Although a genetically determined repolarization abnormality may be important in the initiation of TdP, the sympathovagal imbalance could be a modulating factor. Because the QT interval is continuously modulated by changes in autonomic nervous activity, measurement of the static QT interval may be inadequate for evaluating the risk of TdP in long-QT syndrome.

Recent advances in gene analysis have revealed a genetic heterogeneity in the long-QT syndrome with different loci for this inherited disorder and the relationship of genotype and ECG phenotype in long-QT syndrome has been demonstrated 14,15 It is also possible that the relation between HR and the QT interval is not uniform in long-QT syndrome, ${ }^{5,16,17}$ Although the present study was limited by the absence of gene analysis, the findings provide an insight into the autonomic modulation of the QT interval in patients with long-QT syndrome with respect to initiation of TdP.

\section{References}

1. Moss AJ, Schwartz PJ, Crampton RS, Tzivoni D, Locati EH, MacCluer J, et al: The long QT syndrome: Prospective longitudinal study of 328 families. Circulation 1991; 84: 1136-1144

2. Zipes DP: The long QT syndrome: A rosetta stone for sympathetic related ventricular tachyarrhythmias. Circulation 1991; 84: 14141419

3. Viskin S, Alla SR, Barron HV, Heller K, Saxon L, Kitzis I, et al: Mode of onset of torsade de pointes in congenital long QT syndrome. J Am Coll Cardiol 1996; 28: 1262-1268

4. Locati EH, Maison-Blanche P, Dejode P, Cauchemez B, Coumel P: Spontaneous sequences of onset of torsade de pointes in patients with acquired prolonged repolarization: A quantitative analysis of Holter recordings. J Am Coll Cardiol 1995; 25: 1564-1575

5. Wilde AAM, Jongbloed RJE, Doevendans PA, Duren DR, Hauer RNW, Langen IM, et al: Auditory stimuli as a trigger for arrhythmic events differentiate HERG-related (LQT2) patients from KVLQT1related patients (LQT1). J Am Coll Cardiol 1999; 33: 327-332

6. Shimono M, Fujiki A, Inoue H: Relation between autonomic nerve activity and QT interval in patients with congenital long QT syndrome: Analysis using 24-hour Holter. Ann Noninv Electrocardiol 1998; 3: $12-19$

7. Coumel P: Cardiac arrhythmias and the autonomic nervous system. $J$ Cardiovasc Electrophysiol 1993; 4: 338-355

8. Litovsky SH, Antzelevitch C: Differences in the electrophysiological responses of canine subendocardium and subepicardium to acetylcholine and isoproterenol: A direct effect of acetylcholine in ventricular myocardium. Circ Res 1990; 67: 615-627

9. Ahnve S, Vallin H: Influence of heart rate and inhibition of autonomic tone of the QT interval. Circulation 1982; 65: 435-439

10. Gilmour RF, Riccio ML, Locati EH, Maison-Blanche P, Coumel P, Schwartz PJ: Time- and rate-dependent alterations of the QT interval precede the onset of torsade de pointes in patients with acquired QT prolongation. J Am Coll Cardiol 1997; 30: 209-217

11. Inoue H, Zipes DP: Changes in atrial and ventricular refractoriness and in atrioventricular nodal conduction produced by combination of vagal and sympathetic stimulation that result in a constant spontaneous cycle length. Circ Res 1987; 60: 942-951

12. Fujiki A, Masuda A, Inoue H: Effects of unilateral stellate ganglion block on the spectral characteristics of heart rate variability. Jpn Circ $J$ 1999; 63: $854-858$

13. Schwartz PJ, Priori SG, Spazzolini C, Moss AJ, Vincent GM, Napolitano C, et al: Genotype-phenotype correlation in the long-QT syndrome: Specific triggers for life-threatening arrhythmias. Circulation 2001; 103: 89-95

14. Moss AJ, Zareba W, Benhorin J, Locati EH, Hall WJ, Robinson JL, et al: ECG T-wave patterns in genetically distinct forms of the hereditary long QT syndrome. Circulation 1995; 92: 2929-2934

15. Zhang L, Timothy KW, Vincent M, Lehmann MH, Fox J, Giuli LC, et al: Spectrum of ST-T-wave patterns and repolarization parameters in congenital long-QT syndrome: ECG findings identify genotypes. Circulation 2000; 102: 2849-2855

16. Schwartz PJ, Priori SG, Locati EM, Napolitano C, Cantù F, Towbin AJ, et al: Long QT syndrome patients with mutations of the SCN5A and HERG genes have differential responses to $\mathrm{Na}$ channel blockade and to increase heart rate; implications for gene-specific therapy. Circulation 1995; 92: 3381 -3386

17. Compton SJ, Lux RL, Ramsey MR, Strelich KT, Sanguinetti MC, Green LS, et al: Genetically defined therapy of inherited long-QT syndrome correction of abnormal depolarization by potassium. Circulation 1996; 94: 1018-1022 